\title{
Double layer repair of tibialis anterior muscle hernia in a soccer player: a case report and review of the literature
}

\author{
Gürhan Dönmez ${ }^{1}$ \\ Mustafa Kürsat Evrenos ${ }^{2}$ \\ Meryem Cereb ${ }^{3}$ \\ Yigitcan Karanfil' \\ Mahmut Nedim Doral ${ }^{1}$ \\ 1 Department of Sports Medicine, Hacettepe Univer- \\ sity, Ankara, Turkey \\ 2 Department of Plastic and Reconstructive Surgery, \\ SehitKamil State Hospital, Gaziantep, Turkey \\ 3 Department of Radiology, State Hospital, Gaziantep, \\ Turkey
}

Corresponding author:

Gürhan Dönmez

Department of Sports Medicine, Hacettepe University 06100 Ankara, Turkey

E-mail: gurhan.donmez@hacettepe.edu.tr

\section{Summary}

Background: muscle herniations usually present in athletes especially in the lower legs; occurring through defects in the deep fascial layer of the muscles and typically seen following local blunt trauma or muscle hypertrophy after strenuous exercise. Management of muscle hernias varies from conservative therapy to surgical repair and usually needs multidisciplinary collaboration for differential diagnosis.

Methods: herein tibialis anterior muscle hernia in 17-year-old male soccer player was presented. The diagnosis was confirmed with dynamic ultrasonographic views changing with the different movements of the ankle. Since the symptoms were not relieved with conservative methods, surgical repair of the defect was offered.

Results: we preferred to repair fascial defect with double layer and Mesh graft that were placed over primary suture repair. No complications were reported such as wound or mesh infection postoperatively. The patient was clinically satisfied and returned his previous activity level after 3 months of surgery. After 2 years of follow-up the feature of the bulge was dissolved and player was satisfied with the operation. Conclusion: knowledge of the lower extremity muscle herniation is essential for both proper management and/or surgical referral. The importance of protective devices in prevention, dynamic ultrasonography in diagnosis and double layer repair of the fascial defect with Mesh graft in treatment of muscle herniations were highlighted.

KEY WORDS: mesh graft, muscle herniation, shin guard, soccer, ultrasonography.

\section{Introduction}

Muscle herniations of the lower legs usually present in athletes, soldiers, and in professions requiring excessive strain on the legs. It is common in sports medicine practice patients may seen in dermatology, plastic surgery or general surgery clinics and this has been infrequently reported in sports medicine literature ${ }^{1-3}$. They occur through defects in the deep fascial layer of the muscles. Most hernias occur in the lower leg and tibialis anterior is the common site of herniation ${ }^{4}$. The other possible sites are posterior tibial compartment, peroneal compartment, and quadriceps fascia. These defects in athletes are typically seen following local blunt trauma or muscle hypertrophy after strenuous exercise.

The diagnosis of muscle hernia has classically relied on clinical symptoms and physical examination. Diagnosis should be considered in patients with positionally variable subcutaneous nodules. Typically, a palpable soft-tissue mass is found. The fascial defect can often be palpated. Positioning of the patient's leg with dorsiflexion ("fencer's lunge" position) accentuates the fascial defect for tibialis anterior muscle. Patients present with a swelling that usually appears or enlarges when the patient is standing erect or the affected muscle is contracted. The swelling is disappeared or shrinks when the muscle is relaxed or the patient is supine. Radiologic imaging techniques, including MRI, CT and USG have been used to identify the fascial defect. Although MRI has been reported to be useful in doubtful cases, dynamic USG usually is sufficient for muscle herniations ${ }^{5,6}$.

Treatment depends on the symptoms and varies from conservative therapy to surgical repair. Available surgical procedures were reported as direct repair, fascial grafting, fasciotomy and more recently mesh grafting ${ }^{7}$. Herein, we present a soccer player having tibialis anterior muscle hernia after trauma that was succesfully treated with double layer repair of fascial defect. This case report and literature overview was 
conducted in accordance with international standards and ethical standards ${ }^{8}$.

\section{Case report}

A 17-year-old male soccer player was applied to sports medicine clinic with a resistant swelling in his right leg, which is painful especially during training. He reported a direct trauma of his opponent's shoe cleat 5 months ago in practice and previously treated with activity limitation and analgesics. Clinical investigations revealed a soft tissue mass about 3.5 centimeters diameter in anterolateral aspect of right leg, which was noticeable, both at foot in neutral and tiptoe positions (Fig. 1). However the lesion was disappeared with the patient in supine position. It was painless at rest but had tenderness with palpation. Neurovascular examination of the lower leg was completely normal. His medical history was otherwise noncontributory.

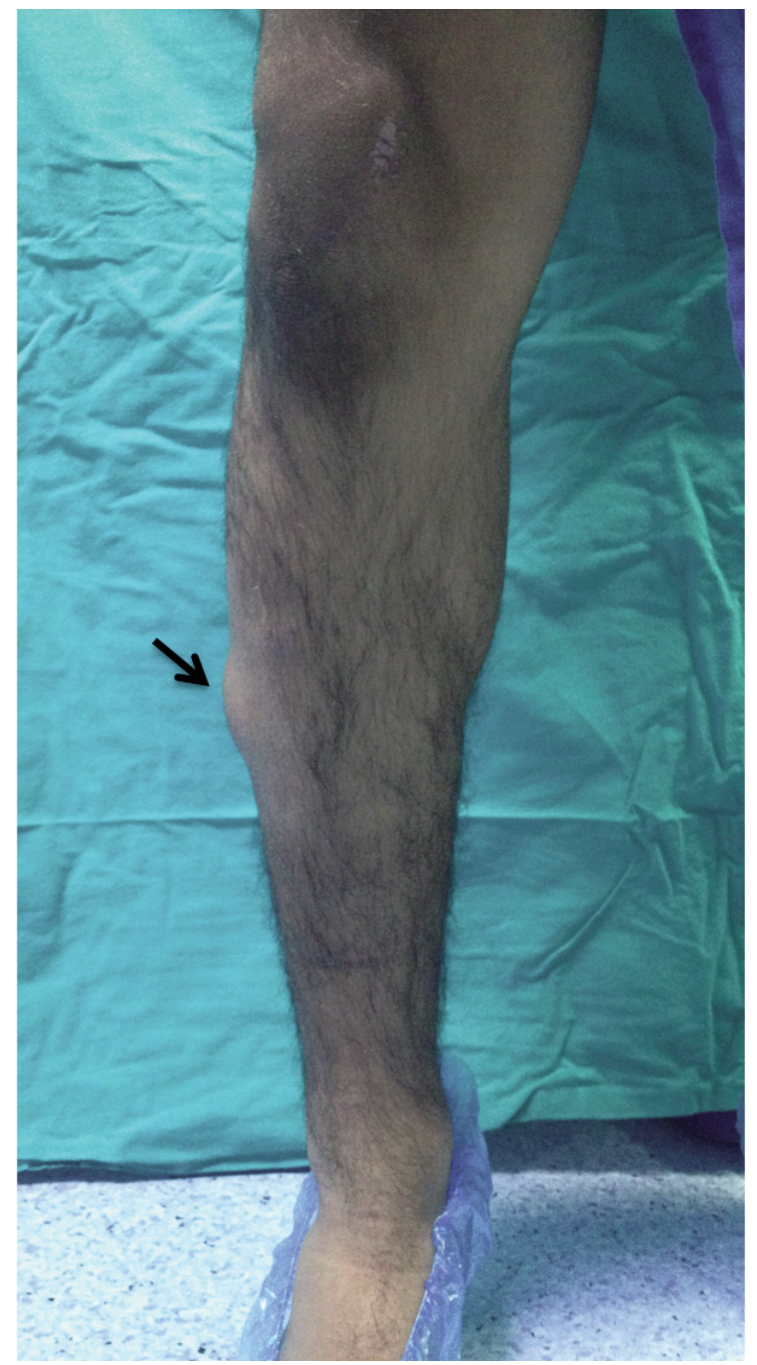

Figure 1. Gross appearance of the protruding mass on the anterolateral aspect of the right leg.
After physical examination, a fascial defect and muscle herniation was suspected and the patient was evaluated with dynamic ultrasonography (USG). UItrasonography of foot in neutral position revealed protrusion of the anterior tibial muscle through the fascia (Fig. 2) and the diagnosis was confirmed with pronounced bulging in dorsiflexion of the ankle. Since the symptoms were not relieved with resting and conservative methods, surgical repair of the defect was offered. The operation was performed under spinal anesthesia with the patient supine. A longitudinal skin incision was made directly above the mass and both fascia borders were exposed (Fig. 3). $3 \mathrm{~cm}$ long longitudinal fascia laceration was examined and both fascia borders were weak. There was no compartment syndrome sign. Double layer repair was administered with 2.0 polydioxanone primary sutures and subsequently $3 \times 5 \mathrm{~cm}$ long polypropylene mesh graft over the primary suture (Fig. 4). 3.0 polypropylene sutures were used to fix mesh graft. Then skin closure was performed with subcuticular sutures. No complications were reported such as wound or mesh infection postoperatively. Short leg casting was applied for two weeks after surgery. Thereafter rehabilitation program including stretching exercises combined with strengthening of anterior compartment was

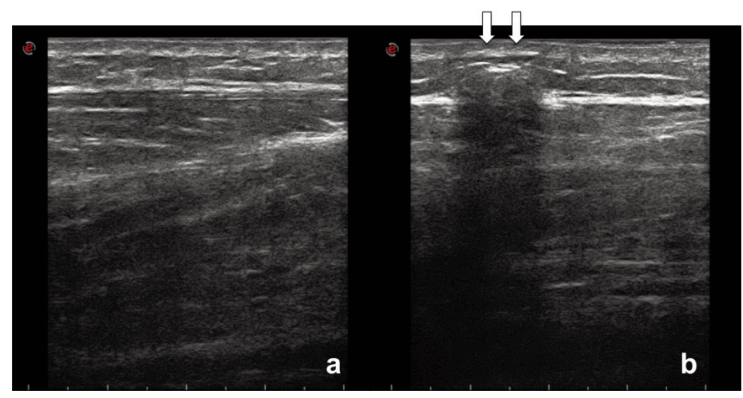

Figure 2. Dynamic ultrasonography shows the loss of continuity of the fascia (b) over the tibialis anterior muscle with contraction.

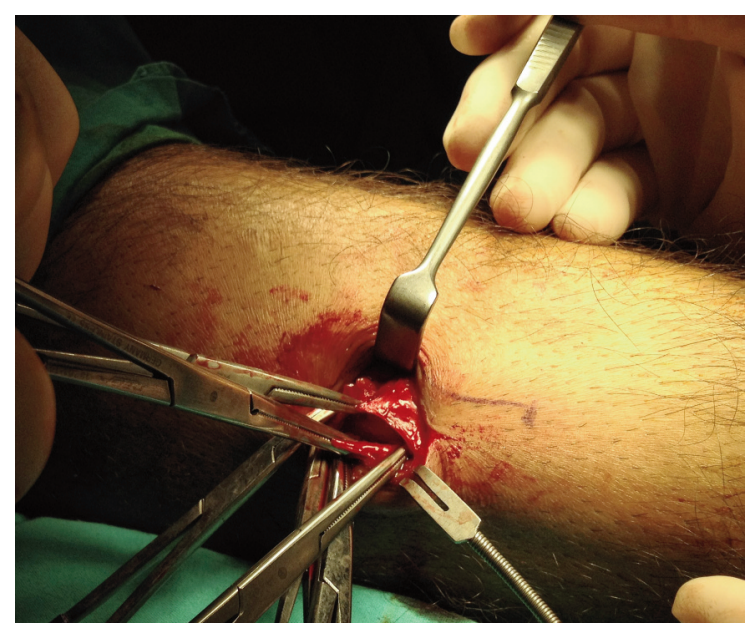

Figure 3. Fascial defect during surgery was detected as 3 $\mathrm{cm}$ diameter. 


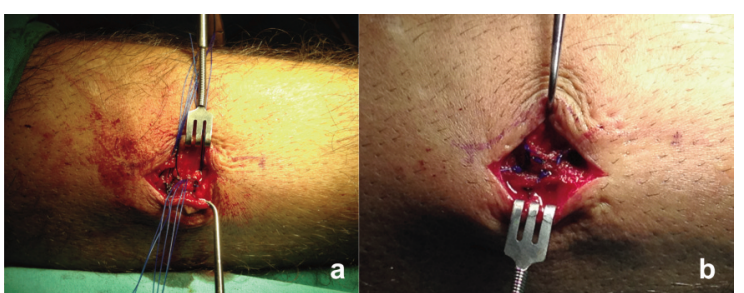

Figure 4. Primary repair (a) and Mesh graft (b) over primary suture.

started at 3th postoperative week. The patient was allowed moderate running activities following 6 weeks of surgery with support hose. 12 weeks later his pain has subsided significantly and he returned her previous activity level without any complaint. After 2 years of follow-up the feature of the bulge was dissolved and player was satisfied with the operation.

\section{Discussion}

Muscle herniations of the lower legs are not uncommon in sports medicine practice; however this entity has been rarely reported and commonly neglected by the physicians ${ }^{2}$. These defects typically occur following local blunt trauma or muscle hypertrophy. Therefore elite athletes tend to face with a muscle hernia during their careers more than any individual. Awareness of these defects is necessary to avoid misdiagnosis and delayed treatment especially in athletes. Muscle hernias have been classified into traumatic and constitutional origin 9-11. Anterior tibial muscle is the most commonly affected muscle of the lower extremities because its fascia is the most vulnerable to trauma. Our patient was a typical case of trauma based tibialis anterior hernia and caused by a direct trauma of opponent's shoe cleat. They are usually caused by a defect in the deep fascial layer and direct trauma leads muscle to protrude through a defect in the fascia into the adjacent subcutaneous fat ${ }^{8}$. Using protective shin guards even in practice sessions may play an important role on preventing these injuries.

Patients usually seek medical advice for pain, cosmetic reasons, or concern of a tumor. Treatment depends on the symptomatology of the herniation and varies from conservative therapy to surgical repair. Asymptomatic hernias require no treatment. Because of possible surgical complications such as compartment syndrome, cosmetic reasons are usually not an indication for surgery ${ }^{12,13}$. For mild cases, symptomatic herniations (cramping, aching pain, exertional discomfort and tenderness) should be initially managed with support hose and can be of benefit along with rest and activity modification. If symptoms persist despite ongoing treatment and conservative treatment fails to alleviate symptoms, an operative treatment should be considered ${ }^{14}$. Available surgical procedures were reported as direct repair ${ }^{15}$, fascial grafting $^{16}$, fasciotomy ${ }^{7,17}$, partial muscular excision and more recently mesh grafting ${ }^{18,19}$. Direct repair is possible when the defect is small and the laxity of the borders permits approximation. However, muscle herniation, particularly of the anterior compartment, is generally not repaired by fascial closure because of the potential for ensuing anterior compartment syndrome ${ }^{12,15}$. Likewise, fascial grafts and side-to-side closures are not recommended, as these may lead to compartment syndrome. Longitudinal fasciotomies were recommended as the treatment of choice in symptomatic tibialis anterior muscle hernias that are refractory to conservative treatment ${ }^{20,21}$. Hedge recommended closure with autologous fascia lata graft or fasciotomy to relieve the symptoms ${ }^{22}$. Although fasciotomy is an acceptable treatment option for this condition, the results are more likely to be cosmetically displeasing. After fascial splitting, adhesions can develop between the muscle and the overlying scar causing a visible deformity when the muscle contracts $^{21}$. Kramer et al. reported that residual symptoms are common especially in runners and despite the satisfactory results with fasciotomy, patients with postsurgical muscle herniations may have the worst clinical outcome ${ }^{7}$.

More recently, repair with synthetic patches has been implemented ${ }^{12,13}$. Marić et al. offered periosteal patch plasty as a possible solution with the properties of easy approachable, cheap and autologous material for anterior tibial muscle hernia treatment ${ }^{23}$. Fascial defect coverage using artificial meshes is simple, more rapid, and less complicated than other techniques and can be used for large defects. Lee et al. ${ }^{4}$ recommended the use of mesh patches for the repair of larger defects and direct closure for only very small, limited defects with close postoperative observation for compartment syndrome. They highlighted that these hernias may be prone to recurrence after surgical repair. However, Marques et al. ${ }^{19}$ reported no recurrence of the multiple fascial defects that were closed with Marlex mesh after 5 years follow-up. In accordance, Siliprandi et al. reported excellent functional results and a good cosmetic appearance with polyester mesh (Mersilene) fixing to the edges of the defect for large anterior tibialis muscle hernias ${ }^{18}$. However the patient required an in patient stay of 7 days and was restricted in weight bearing for 2 weeks with this technique. The use of a polypropylene mesh ensured succesful repair of iatrogenic thigh hernias without major decrease in compartmental volume ${ }^{24}$. Nochëvkin and Illarionov reported good anatomofunctional and cosmetic results in 5 cases with method of plastic closing the fascial defects with the polymer tissue 25 .

Since the patient in our case was young and planning high level competition in near future, also reason of defect was direct trauma and fascia borders were weak, we preferred to repair fascial defect with double layer and Mesh graft that were placed over primary suture repair. The patient was clinically satisfied and returned his previous activity level after 3 months of surgery. We highlighted to sports medicine surgeons thay they always should be kept in 
mind for the recurrence of these defects and double layer repair may decrease the risk of recurrence of muscle herniations.

In conclusion, the knowledge of the lower extremity muscle herniation is essential for both proper management and/or surgical referral. Despite the first option in treatment should be conservative approach, surgical options should be considered if the symptoms persist and lead limitations in athletic performance. Double layer repair may be promising technique in order to prevent re-injuries.

\section{References}

1. Berglund HT, Stocks GW. Muscle hernia in a recreational athlete. Orthop Rev. 1993;22:1246-1248.

2. Gupta RK, Singh D, Kansay R, Singh H. Cricket ball injury: a cause of symptomatic muscle hernia of the leg. Br J Sports Med. 2008;42;1002-1003.

3. Ceyhan AM, Chen W, Yener M, Yildirim M, Yesildag A, Akkaya VB. Bilateral tibialis anterior muscle herniation simulating a soft tissue tumour in a young amateur football player. Australas $\mathrm{J}$ Dermatol. 2010;51:142-144.

4. Lee HS, Kim MJ. Painful bilateral herniation of the anterior tibial muscle: a case report. Foot \& Ankle International. 2006; 27(7):552-555.

5. Bates DG. Dynamic ultrasound findings of bilateral anterior tibialis muscle herniation in a pediatric patient. Pediatr Radiol. 2001;31:753-755.

6. Beggs I. Sonography of muscle hernias. Am J Radiol. 2003; 180:395-399.

7. Kramer DE, Pace JL, Jarrett DY, Zurakowski D, Kocher MS, Micheli LJ. Diagnosis and management of symptomatic muscle herniation of the extremities: a retrospective review. Am J Sports Med. 2013;41(9):2174-2180.

8. Padulo J, Oliva F, Frizziero A, Maffulli N. Muscles, Ligaments and Tendons Journal. Basic principles and recommendations in clinical and field science research. MLTJ. 2013;4:250-252.

9. Bergmann G, Ciritsis BD, Wanner GA, Simmen HP, Werner $\mathrm{CM}$, Osterhoff $\mathrm{G}$. Gastrocnemius muscle herniation as a rare differential diagnosis of ankle sprain. Patient Saf Surg. 2012; 14;6(1):5.
10. Angadi DS, Rampaul RS, Akthar I, Makdhoomi K. Bilateral muscle hernias of the anterior tibial muscle. Foot Ankle Int. 2007;28(4):520.

11. Kim M, Hong SP, Hwang SM, Park H, Ahn SK. Tibialis anterior muscle herniation developed after trauma. Int J Dermatol. 2008:47:845-847.

12. Miniaci $\mathrm{A}$, Rorabeck $\mathrm{CH}$. Compartment syndrome as a complication of repair of a hernia of the tibialis anterior. J Bone Joint Surg. 1986;68:1444-1445.

13. Wolfort $\mathrm{F}$, Mogelvang $\mathrm{C}$, Filtzer $\mathrm{H}$. Anterior tibial compartment syndrome following muscle hernia repair. Arch Surg. 1973; 106:97-99.

14. Lane JE, Woody CM, Lesher JL. Tibialis anterior muscle herniation. Dermatol Surg. 2002;28:641-642.

15. Williams DP, Hassan Al. Undiagnosed compartment syndrome following anterior tibialis muscle hernia repair. Injury Extra. 2007;38:59-60.

16. Hartzell J. The use of living fascia transplant to repair a hernia of the tibialis anticus muscle. J Am Med Assoc. 1936;107:492493.

17. Miniaci $\mathrm{A}$, Rorabeck $\mathrm{CH}$. Tibialis anterior muscle hernia: a rationale for treatment. Can J Surg. 1987;30:79-80.

18. Siliprandi L, Martini G, Chiarelli A, Mazzoleni F. Surgical repair of an anterior tibialis muscle hernia with Mersilene mesh. Plast Reconstr Surg. 1993;91:154-157.

19. Marques A, Brenda E, Amarante MT. Bilateral multiple muscle hernias of the leg repaired with Marlex mesh. Br J Plast Surg. 1994;47:444-446.

20. Bloem JJ. The treatment of muscle hernias by fascial splitting Br J Plast Surg. 1976;29(4):291-294.

21. Lewis JR, Shaw A, Arrowsmith J, Stephen AB. The symptomatic tibialis anterior hernia: Case report and a new rationale for treatment. Injury Extra. 2008;39:4-6.

22. Hegde AS. An interesting case of post traumatic tibialis anterior muscle herniation. Kathmandu Univ Med J (KUMJ). 2013; 11(44):332-334.

23. Marić $\mathrm{D}$, Madić $\mathrm{D}$, Marić $\mathrm{D}$, Stanković $\mathrm{M}$, Smajić M. Anterior tibial muscle hernia-reconstruction with periosteal patch plasty. [Article in Serbian] Vojnosanit Pregl. 2009;66(12):10151018.

24. Richards $\mathrm{H}$, Thomas R, Upadhyay SS. Polypropylene mesh repair of iatrogenic thigh hernias. Injury. 1998;29(6):478.

25. Nochëvkin VA, Illarionov VV. Surgical treatment of true muscle hernias of the extremities. [Article in Russian] Vestn Khir Im I I Grek. 1999;158(3):70-71. 